\title{
EL TRATADO ASTRONÓMICO DEL SEXAGENARIUM: UNA APORTACIÓN MUDÉJAR VALENCIANA A LA HISTORIA DE LA CIENCIA ${ }^{1}$
}

\author{
Maravillas Aguiar Aguilar* \\ José Antonio González Marrero*
}

\section{INTRODUCCIÓN:}

\author{
LA ESPAÑA MUDÉJAR Y LA PERVIVENCIA DE LA LENGUA \\ ÁRABE COMO LENGUA DE COMUNICACIÓN CIENTÍFICA. LA \\ INTRODUCCIÓN DEL SEXAGENARIUM EN OCCIDENTE
}

En los territorios de la Península Ibérica que aún eran musulmanes vivían cristianos a los que se les respetaban sus costumbres y cultos, siempre y cuando no hicieran ostentación de ello ${ }^{2}$. En los territorios ya conquistados por los cristianos se permitió, mediante distintos acuerdos que fueron sucediéndose en el tiempo, que los musulmanes siguieran profesando su fe. Esos musulmanes a los que se les había permitido quedarse eran los mudéjares -del árabe mudâŷyan-. El Llibre de la Çuna e Xara dels Moros ${ }^{3}$ atestigua la coexistencia en el antiguo reino de Valencia, y tras la conquista de este reino por Jaime I (siglo XIII), del derecho islámico y de otras normas legales como los Furs de Valencia, el Fuero de Aragón, los Usatges de Barcelona y la Costum

Facultad de Filología. Universidad de La Laguna.

1. Los autores de este trabajo quieren agradecer de forma muy especial los comentarios, correcciones y sugerencias que los profesores Míkel de Epalza y Julio Samsó han hecho tras la lectura de sucesivos borradores, mejorando notablemente estas páginas.

2. Una visión general acerca de los mudéjares y moriscos se encuentra en M. de Epalza, Los moriscos antes y después de la expulsión, Madrid, 1992.

3. Vid. C. BARCELO, Un tratado catalán de derecho islámico: El Llibre de la Çuna e Xara dels Moros, Córdoba, 1989. 
d'Espanya, sólo aplicada esta última a la tenencia de los castillos. A partir de 1329, los Furs de Valencia fueron implantándose como única norma jurídica cristiana, pero otorgándose al mismo tiempo al derecho islámico un respaldo legal que no había tenido antes y que mantendría hasta $1525^{4}$. Mediante las capitulaciones de Santa Fe (Granada, 17 de abril de 1492) los musulmanes que quisieran continuar viviendo en España podían conservar sus mezquitas con sus alminares, permitiéndose la llamada a la oración, se les respetaban sus ritos, no se les secuestrarían sus bienes habuses ${ }^{5}$, la justicia entre musulmanes sería ejercida por cadíes y de acuerdo con el derecho islámico, los alfaquíes y ulemas podían seguir enseñando a sus fieles el dogma de la fe islámica. El arzobispo de Granada, Hernando de Talavera, aprendió árabe y mandó traducir a esta lengua muchos libros para poner al alcance de los mudéjares la doctrina cristiana. Este clima de tolerancia quedó roto con la llegada a Granada en 1499 del confesor de la reina Isabel y arzobispo de Toledo, Francisco de Cisneros (1436-1517), que sustituiría al arzobispo Hernando de Talavera (m. 1507). Una quema de libros junto a la puerta de Bibarrambla fue una de sus acciones. Su política de cristianización forzosa culminó con el decreto de expulsión de mudéjares de 1501, año a partir del cual todo musulmán en territorio español debía convertirse al cristianismo o ser deportado. Los que siguieron el camino de la conversión forzosa dieron lugar a un nuevo grupo dentro de la sociedad española, los moriscos, musulmanes que habían abrazado la fe cristiana más o menos forzadamente. Aquellos musulmanes españoles que se negaron a convertirse al cristianismo fueron deportados. Un siglo después, en 1609, Felipe III dio orden de expulsar a todos los moriscos. La expulsión culminó hacia 1614 y en algunas regiones peninsulares, como la valenciana y la aragonesa, supuso una verdadera catástrofe económica, si bien el caso de estas regiones no es extensivo a toda España.

En un contexto mudéjar, no plenamente islámico, debemos encuadrar el sexagenarium. La presencia islámica en la transmisión y comunicación científicas durante la segunda mitad del siglo XV tiene su más vivo reflejo entre los alfaquíes valencianos ${ }^{6}$. En el año 1450 y procedente de El Cairo, un alfa-

4. Ibidem, p. X. Acerca del carácter oficial del derecho islámico en territorio valenciano véase C. BArCelo, Minoriás islámicas en el País Valenciano. Historia y dialecto, Valencia, 1984, p. 57 y ss.

5. En castellano se aceptan los términos habús y habiz, plural habuses. Su origen es árabe, de hubus, plural alıbās. El habús o habiz era una renta procedente de fundaciones que servía para sostener mezquitas, madrasas u otras actividades islámicas de uso público.

6 Cf. L. GARCÍA BALLESTER, Historia social de la medicina en la España de los siglos XllI al XVI, Madrid, 1976, vol. I, p. 72, en la que citamos textualmente “... nos habla del vigor y autonomía científica de concretos grupos de moros valencianos, del mantenimiento de la actividad científica por parte de los alfaquíes, de la comunicación con el mundo islámico oriental y de la atención que determinados médicos y astrónomos cristianos, pertenecientes a la selecta minoría docente, prestaban a la interesante ciencia que se hacía en países como Egipto, y practicada por mudéjares del país". 
quí de Paterna introdujo en Valencia un instrumento astronómico conocido con el nombre latino de sexagenarium ${ }^{7}$, que tiene relación con la familia de los ecuatorios, aunque su uso resulta muy complejo ${ }^{8}$.

El caso del sexagenarium es particularmente interesante ya que se trata de un instrumento utilizado por astrónomos de El Cairo y conservado en dos manuscritos ${ }^{9}$. También se hizo, al parecer, un pequeño comentario impreso a principios del siglo XVI. Además, se conserva actualmente en el Museo de Historia de la Ciencia de Oxford ${ }^{10}$ un ejemplar de latón que proviene del gabinete de objetos curiosos legado, en el siglo XVIII, por el coleccionista John Pointer al St-John College de Oxford ${ }^{11}$.

La traducción al valenciano y al latín, y quizás al italiano ${ }^{12}$, del tratado árabe que describía dicho instrumento es uno de los últimos casos conocidos de transmisión científica a través de España ${ }^{13}$.

\section{EL SEXAGENARIUM}

Tradicionalmente, la crítica considera el sexagenarium un instrumento de origen árabe de la familia de los ecuatorios. Es un objeto plano en forma

7. Esta relación aparece en primer lugar en E. POULLE, «Théorie des planètes et trigonométrie au XV siècle, d'après un équatoire inédit, le sexagenarium", Journal de's Savants, 3 (1966), pp. 129-161, y después en varios trabajos posteriores.

8. A pesar de las presiones a las que fue sometido este alfaquí no permitió a nadie conocer el contenido de este tratado hasta su muerte. Sin duda alguna, el instrumento debía ayudarle a resolver cuestiones relacionadas con el ritual islámico, un secreto que le convenía guardar bien en la comunidad musulmana de mediados del siglo XV que residía en Valencia. Su desconfianza revela, por otro lado, la crisis de convivencia entre la minoría mudéjar y la cristiana en esta ciudad. En otra ocasión ahondaremos en dos de los grandes disturbios contra musulmanes acaecidos en Valencia, en 1391 y 1455 respectivamente.

9. Vid. en el tercer apartado de este estudio.

10. Objeto n. ${ }^{\circ} 59$.

11. Cf. E. Poulle, op. cit., p. 133 y «Sexagenarium», en Les instruments de la théorie des planètes selon Ptolémée: Equatoires et horlogérie planétaire du XIII au XVI siècle, Genève-Paris, 1980, p. 419.

12. Véase L. THORNDIKE, «Sexagenarium», Isis, 42 (1951), pp. 130-133, donde describe el contenido de los manuscritos de París y transcribe los títulos de sus capítulos. En el mismo trabajo se sugieren dos puntos, a nuestro entender, erróneos: a) el autor creía que la versión en lengua valenciana era un texto en español ( $c f$. p. 130) y b) creía posible la existencia de una versión italiana (ibidem), ¿quizás tras la lectura de esta frase del folio 57v del manuscrito 7416 A?: "Incipit lectura sexagenarium quam Johannes bonie ex arabico in ydioma ualentinum interpretatus est et quidam in romanam linguam omnium preclarissimam nunc traduxit" . Anteriormente, el mismo Thorndike habia hecho una breve mención al sexagenarium en «Pre-copernican astronomical activity», Proceedings of the American Philosophical Society, XCIV (1950), pp. 321-326.

13. Cf. J. SAmso, Las ciencias de los antiguos en al-Andalus, Madrid, 1992, p. 390. 
de cuarto de círculo que presenta en cada una de sus dos caras un trazado diferente. Ambos trazados se utilizaban en combinación uno con el otro. Una de las caras, ocupada por una especie de ecuatorio, viene a ser la denominada "cara planetaria" del instrumento. Dicha cara lleva un disco con quince círculos concéntricos y sobre él una regleta móvil. La otra cara del sexagenarium Ileva inscrito un cuadrante de $\operatorname{senos}^{14}$. Veamos separadamente cada uno de estos componentes.

\section{El ECUATORIO ${ }^{15}$}

Etimológicamente el término ecuatorio proviene de la palabra latina equatorium, del verbo latino equare ${ }^{16}$. Este verbo tenía durante la Edad Media entre sus acepciones la de "calcular la ecuación de un planeta", es decir, calcular la corrección que debe aplicarse a las coordenadas angulares medias del planeta para conocer su longitud verdadera. La lengua árabe utilizó generalmente en su terminología astronómica medieval el verbo ta'addala con la misma acepción y la palabra $t a^{c} d \tau l$ con la significación de "ecuación".

El ecuatorio es un instrumento plano construido para reproducir gráficamente el movimiento de los planetas. Mediante el uso del ecuatorio se evitaba tener que realizar una serie de cálculos largos y complicados. El ecuatorio es pues un modelo planetario a escala que permite resolver un problema concreto, el cálculo de la ecuación de un planeta, de aplicación fundamentalmente didáctica y astrológica.

Ptolomeo evidencia de forma implícita en sus Tablas manuales la existencia de instrumentos planetarios que reproducían geométricamente la posición real de los planetas. Pero estos instrumentos planetarios tenían una finalidad distinta a la que tenían los ecuatorios propiamente dichos, ya que servían para mostrar la mecánica celeste y no para calcular la ecuación de los planetas. Estos últimos aparecieron por vez primera en al-Andalus. Frente al pequeño número de ejemplares que se han conservado hasta nuestros días, tenemos un gran número de tratados que describen cómo se fabrica el ecuatorio y cómo se utiliza. Los tratados sobre el ecuatorio más antiguos que se

14. El único ejemplar que, según Poulle, existe de sexagenarium, se conserva actualmente en el Museo de Historia de la Ciencia de Oxford.

15. Vid. E. Poulle, Les instruments de la théorie des planètes selon Ptolémée: Equatoires et Horlogerie Planétaire du XIITe au XVI siècle, 2 vols., Genève-Paris, 1980. La última monografía aparecida dedicada a los ecuatorios andalusíes es de M. COMES, Ecuatorios andalusies. Ibn al-Samh, al-Zargälluh y Abü-l-Salt (Anuari de Filologia, vol. XIII, 1990, Secció B, Número 1), Barceloná, 1991. En este apartado nos basamos en ella, especialmente en las páginas 17 a 20.

16. Cf. Thesaurus Linguae Latinae, Lipsiae, 1903, vol. I, fasc. V, p. 1006 y pp. 1018-1028. 
conservan son del siglo XI y XII, y sus autores fueron los astrónomos andalusíes Ibn al-Samh ${ }^{17}$, Azarquiel ${ }^{18}$ y Abũ-l-Salt ${ }^{19}$.

El ecuatorio llegó probablemente a Oriente procedente de la España musulmana. Tenemos que remontarnos hasta el siglo XV para encontrar el tratado sobre el ecuatorio de al-Kāšî ${ }^{20}$ como eslabón siguiente en la tradición árabe del instrumentro. Este tratado parece ser el último de los de su especie $^{21}$.

Mientras tanto, la Europa latina desarrolló el instrumento. Conviene reseñar la existencia ya en el siglo XIII de los ecuatorios de Campanus de Novara $^{22}$ y el de Henri Bate de Malines ${ }^{23}$. En el siglo XIV encontramos el tra-

17. Matemático y astrónomo originario de Córdoba que vivió entre 979 y 1035 . Fue discípulo de otro gran astrónomo, Maslama de Madrid (m. 1008). El tratado de astrolabio de Ibn alSamh está traducido y estudiado en M. VILADRICH i GRAU, El "Kitāb al-camal bi-l-asturlāb" (Llibre de l'ús de l'astrolabi d'Ibn al-Samh)). Estudi $i$ Traducció, Barcelona, 1986. Para el nombre, una pequeña reseña biográfica del autor y repertorio de sus obras conservadas véase $C$. BROCKELMMAN, Geschichte der Arabischen Litteratur, 2 vols. (Weimar, 1898), Berlin, 1902, vol. I, p. 472. Reseña biobibliográfica también en M. COMEs, op. cit., pp. 28-29 así como el análisis del tratado del ecuatorio en pp. 32-78.

18. Abū Ishāq Ibrāhīm Ibn Yahyā al-Naqqāš al-Zarqāīi, célebre astrónomo y constructor de instrumentos de observación astronómica, nacido ca. 1030. Desarrolló su actividad en Córdoba, ciudad donde murió el 15 de octubre de 1100. La forma castellana de su nombre que se acepta generalmente es Azarquiel. Sobre Azarquiel hay abundante bibliografía. Véase especialmente J.M. Mrllas Vallicrosa, Estudios sobre Azarquiel, Madrid-Granada, 1943-1950. Los últimos estudios realizados relativos a algunos de sus instrumentos son $\mathrm{R}$. PuIG, Al-šakkāzityya. Edición, traducción y estudio por..., Barcelona, 1986 y R. PUIG AgulLAR, Los tratados de construcción y uso de la azafea de Azarquiel, Madrid, 1987. Sobre su tratado acerca del ecuatorio véase M. CoMEs, op. cit., pp. 79-138.

19. Polígrafo originario de Denia que vivió entre 1067 y 1134 . Fue autor de obras de filosofía, historia, astronomía, medicina, poesía, crítica literaria, música, etc. Véase C. BROCKELMMAN, op. cit., vol. I, pp. 486-487 y Supplementband, 3 vols., Leiden, 1937-1942, vol. I, p. 889. Un resumen de su vida y reseña de sus obras aparece en M. COMES, op. cit., pp. 140-141. EI estudio de su tratado del ecuatorio está en ibidem, pp. 143-157.

20. Yamšì Giyāt al-Dīn al-Kāšī, muerto en 1429. Su tratado acerca del ecuatorio ha sido estudiado en E.S. KENNEDY, "A Fifteenth-Century Planetary Computer: al-Käshi's "Tabaq alManāteq". I. Motion of the Sun and Moon in Longitude», Isis, 41 (1950), pp. 180-183; reimpreso en E.S. KENNEDY, Studies in the Islanic Exact Sciences, Beirut, 1983, pp. 452-455; y en E.S. KENNEDY, «A Fifteenth-Century Planetary Computer: al-Kāshī's "Tabaq al-Manäteq". II. Longitudes, Distances and Equations of the Planets», Isis, 43 (1952), pp. 42-50; reimpreso en E.S. KENNEDY, Studies in the Islamic Exact Sciences, Beirut, 1983, pp. 472-480; y en E.S. KENNEDY, The Planetary Equatorium of Jamshīd Ghiyäth al-Dīn al-Käshĩ, Princeton, 1960.

21. Cf. M. COMEs, op. cit., p. 159.

22. Matemático y astrónomo de origen italiano muerto en 1296 . En su Theorica planetarum incluía las instrucciones necesarias para construir un ecuatorio. Se trata ésta de la primera descripción de un ecuatorio en la Europa latina. La edición crítica, traducción, comentario e introducción de esta obra la realizaron F.S. Benjamin y G.J. Toomer. Cf. G.J. ToOMER, «Campanus of Novara», en Dictionary of Scientific Biography, C.C. Gillispie (ed.), 8 vols., New York, 1981 [en adelante $=D S B$ ], vols. 3 y 4, pp. 23-29.

23. Astrónomo originario de Malines, en la actual Bélgica. Nació en 1246 y murió ca. 1310. E1 ecuatorio ideado por Bate se inscribe en la tradición del de Campanus de Novara. El texto 
tado de Johannes de Lineriis ${ }^{24}$ y el de Geoffrey Chaucer ${ }^{25}$. Jean Fusoris ${ }^{26}$ será el inventor de un ecuatorio que no necesitará el uso de tablas astronómicas accesorias pero el instrumento tenía el inconveniente de estar compuesto por un gran número de láminas. Este escollo fue superado por Guillaume Gilliszoon ${ }^{27}$, a finales del siglo XV, al perfeccionar el ecuatorio de Fusoris. En la tradición occidental latina siguen apareciendo ecuatorios hasta el siglo XVII pero sin aportar novedades destacables.

La denominada cara planetaria del sexagenarium aporta los datos suficientes para la resolución de problemas con la otra cara, la del cuadrante de senos. En su conjunto, no constituye un instrumento de observación astronómica sino un instrumento accesorio de cálculo. El sexagenarium formaría parte del grupo de los ecuatorios, si bien éstos, hasta en su versión más mediocre, ofrecen soluciones mucho más sencillas que las que pueda ofrecer nuestro instrumento ${ }^{28}$.

del tratado del ecuatorio de Bate es muy breve y oscuro a juicio de Poulle (vid. infra) y su edición fue reproducida en R.T. GUNTHER, Astrolabes of the World, Oxford, 1932, vol. II, pp. 368-376. Este tratado estaba, junto a un tratado de astrolabio, en la edición que Bate realizó del De nativitatibus de Abraham Ibn Ezra, aparecida en Venecia en 1485. Posteriormente, en 1491, aparecería una edición que contenía únicamente el De nativitatibus de Ezra, sin que se incluyera ni el tratado de astrolabio ni el del ecuatorio, como había ocurrido en la edición de 1485. Cf. E. Poulle, «Henry Bate of Malines», en DSB, vols. 5 y 6, pp. 272-275.

24. Johannes de Lineriis, astrónomo y matemático fallecido ca. 1355. Autor de un almanaque, de tablas astronómicas y de varios tratados de instrumentos. Entre sus obras sobre construcción y uso de instrumentos astronómicos se encuentran dos tratados acerca del ecuatorio. Uno de ellos es una adaptación del tratado de Campanus de Novara pero el otro es sensiblemente diferente. El primer tratado sobre el ecuatorio que hemos mencionado fue publicado por D.J. Price como un apéndice al tratado atribuido a Chaucer en The Equatorie of the Planetis, Cambridge, 1955, pp. 188-196. Pero a juicio de Poulle este trabajo necesita una revisión. El segundo tratado permanece, al parecer, inédito en los manuscritos Vaticano Urbin. lat. 1399, fols. 16-21, en el que se encuentra la explicación de los usos y construcción del instrumento, y Oxford, Digby 228, fols. $53 \mathrm{v}-54 \mathrm{v}$., en el que sólo aparecen sus usos. Cf. E. POULLE, «John of Lignères», en DSB, vols. 7 y 8, pp. 122-128.

25. Autor de obras literarias entre las que descuellan sus Canterbury tales. Nacido y fallecido en Londres, vivió entre 1343 y 1400, y fue un buen conocedor de la literatura cortesana francesa. Escribió dos obras de tema astronómico pocos años antes de morir, A Treatise on the Astrolabe (1391) y Equatorie of the Planetis (1392). Esta última, acerca del ecuatorio, fue editada por D.J. de SOlLA PRICE, The Equatorie of the Planetis, Cambridge, 1955. Cf. D.J. de SollA PRICE, «Geoffrey Chaucer», en DSB, vols. 3 y 4, pp. 217-218.

26. Astrónomo nacido en Giramount $r$ Francia, ca. 1365 y fallecido en 1436 . Vid. E. Poulle, Un constructeur d'instruments astronomiques an XV siècle: Jean Fusoris, Paris, 1963.

27. Astrólogo y fabricante de relojes y de astrolabios. Su tratado acerca del ecuatorio está estudiado en E. POuLLE, «L'équatoire de Guillaume Gilliszoon de Wissekerke», Physis, 3 (1961), pp. 223-251.

28. Cf. E. PoulLE, «Théorie des planètes...», pp. 133 y 161. 


\section{EL CUADRANTE DE SENOS 29}

Entre los instrumentos astronómicos de origen islámico se encuentra el cuadrante de $\operatorname{senos}^{30}$. Las denominaciones más frecuentes con las que se conoce a este trazado son, en lengua árabe, $r u b^{c} a l-m u \hat{y} a y y a b, r u b^{c} a l-\hat{y} u y \bar{u} b$ y $r u b^{c}$ al-dastūr. En latín se le conoce como quadrans canonis, traducción literal de la expresión árabe $r u b^{c}$ al-dastūr, o quadrans vetustissimus, para distinguirlo del quadrans vetus -o cuadrante horario- y del quadrans novus -o cuadrante astrolabio- ${ }^{31}$. El cuadrante de senos es un trazado plano de carácter universal.

El cuadrante de senos está constituido por una red de líneas verticales y horizontales que parten de los lados rectos del cuadrante. Estas líneas forman una red parecida a la trama de una hoja de papel milimetrado moderno y son paralelas una a una a los lados rectos del cuarto de círculo. No siempre el trazado del cuadrante de senos presenta líneas equidistantes entre sí, si bien en la tradición occidental aparece, en los casos que conocemos, un trazado de líneas rectas que guardan distancias iguales entre sí. Además de esta red, un cuarto de circunferencia va desde un extremo de una de las líneas rectas hasta el extremo de la otra. Ambas rectas son de la misma magnitud ya que son por definición radios del círculo origen. Las líneas que parten de las graduaciones del cuadrante determinan una escala gráfica de senos y cosenos.

La función principal del cuadrante de senos es la de medir ángulos pero su genio reside en ofrecer la posibilidad de resolver gráficamente problemas de trigonometría plana y esférica, estos últimos por aproximación. Sirve además para realizar operaciones aritméticas sencillas.

El cuadrante de senos fue desarrollado en Bagdad en el siglo IX y se mantuvo popular en el oriente islámico durante prácticamente un milenio. Originalmente fue inventado para resolver un problema concreto, la deter-

29. Parte de esta introducción ya ha aparecido en alguno de nuestros trabajos como M. ACUIAR Agullar, «Le quadrant à sinus et la mesure du temps et de I'espace» (inédito); id., "En torno a ${ }^{\mathrm{C}} \mathrm{Izz}$ al-Dinn ${ }^{\mathrm{C}} \mathrm{Abd}$ al-cAzizz b. Mas ${ }^{\mathrm{C}} \overline{\mathrm{u}} \mathrm{d}$ (siglo XIV): vida y reseña de sus obras conocidas», Boletín de la Asociación Española de Orientalistas, XXXII (1996), pp. 187-196; id., «Las aplicaciones del cuadrante de senos en agrimensura a través de un tratado árabe oriental del siglo XIV", en Ciencias de la naturaleza en Al-Andalus. Textos y Estudios IV, C. Álvarez de Morales (ed.), Granada, 1996, pp. 93-113.

30. El repertorio más exhaustivo de instrumentos islámicos es D.A. KING, «Astronomical instrumentation in the Medieval Islamic World». Esta obra se encuentra inédita por el momento y de ella conocemos el pre-print n. ${ }^{\circ} 23$ del Institut für Geschichte der Naturwissenschaften de la Universidad Goethe de Frankfurt am Main.

31. Véase un reciente resumen de los diferentes tipos de instrumentos de uso en astronomía en D.A. KING, «Some remarks on islamic astronomical instruments», Scientiarum Historia, 18 (1992), pp. 5-23. 
minación del tiempo en función de la altura del sol y su altura meridiana mediante una fórmula aproximada. Con el paso del tiempo fue transformándose en una especie de regla de cálculo para los astrónomos medievales ${ }^{32}$. El texto árabe más antiguo relativo al uso del cuadrante de senos fue compuesto en el siglo IX por Abū Ŷ́acfar Muhammad b. Mūsà al-Jwārizmīin ${ }^{33}$.

En el proceso de transmisión y expansión de este instrumento podemos diferenciar dos tradiciones, una islámica y otra no islámica. La tradición islámica es la más rica debido al propio origen del trazado y a la prolongada presencia del Islam en regiones del Magreb y Próximo Oriente, y también debido al frecuente uso del cuadrante de senos en astronomía aplicada a ciertas cuestiones del ritual islámico. En efecto, el cuadrante de senos aparece normalmente en el dorso de los astrolabios de factura oriental, mientras que la tradición hispánica, tanto árabe como latina, de construcción de astrolabios parece ignorarlo ${ }^{34}$. El cuadrante de senos es también un trazado de aparición frecuente en los cuadrantes propiamente dichos. Algunos capítulos relativos al cuadrante de senos se encuentran en una obra inédita de al-Bïrū-

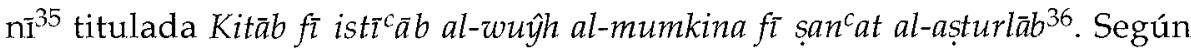
Puig, de ella parecen derivar los tratados posteriores en los que se describe el uso del cuadrante de senos ${ }^{37}$. Por otro lado, la contribución de al-Marrākušĩ (m. 1262) al desarrollo de estos tratados de uso del instrumento está aún por estudiar. Puede ser útil comenzar por el apartado acerca del uso del cuadrante de senos que hay en su Ŷāmic al-mabādi' wa-l-gayāt fi ${ }^{c} i l m$ al-mī qă $t^{38}$ y estudiar su Al-Mujtasar fi kayfĩ yat al-camal bi-l-waŷh al-yaybi min waŷhay rubc al-dustür (Resumen acerca del uso de la cara del cuadrante de senos del cuadrante dustūr $)^{39}$.

Ya del siglo XIV es la Risāla käfiyat al-sayb fî-l-camal bi-l-yayb (Tratado del regalo suficiente para usar el cuadrante de senos) de la que ya nos hemos ocupado en más de una ocasión ${ }^{40}$.

32. Ibidem, p. 17.

33. Matemático y astrónomo que desarrolló su actividad científica en Bagdad. Vivió ca. 800847. Al final de dos tratados de astrolabio incluyó un breve apartado dedicado al cuadrante de senos que se conserva en un manuscrito árabe de la Biblioteca Nacional de Berlín (Deutsche Staatsbibliothek), Ahlwardt n. ${ }^{\circ}$ 5791-5793.

34. Cf. R. Purc, «Instrumentos universales en al-Andalus», en El Legado Cientifico Andalusi, Madrid, 1992, p. 70.

35. Astrónomo y matemático musulmán fallecido en 1048.

36. Cf. R. PulG, «Una aportación andalusí a la difusión del cuadrante de senos», Yãa-Nãma in memoria di Alessandro Bausani, Roma, 1991, II, pp. 79-80.

37. Ibidem, p. 80.

38. lbídem. Según Puig, al-Marrākušī se basó en Bīrūnī.

39. Compuesto por 111 secciones y conservado en Ankara, Biblioteca Ismail Saib, ms. 5111. Ocupa 20 folios. Siglo VIII A.H./ XIV A.D.

40. Este tratado está dedicado monográficamente a la explicación de las distintas aplicaciones del cuadrante de senos. Fue compuesto en El Cairo en el año 1393 y de él parece haberse 
El cuadrante de senos es un trazado, pues, de origen islámico acerca del cual sabemos aún muy poco y acerca del cual hay un gran campo de trabajo abierto al investigador.

\section{ESTABLECIMIENTO DEL CORPUS DE TEXTOS PARA LA EDICIÓN DEL TRATADO DEL SEXAGENARIUM}

El tratado que describe el sexagenarium aparece recogido fundamentalmente en dos manuscritos que se conservan en la Biblioteca Nacional de París ${ }^{41}$. Contamos, en primer lugar, con el manuscrito 7416A. Es el testimonio que posee el mayor número de datos tanto acerca de la obra en sí misma como en torno al autor. Se trata de un misceláneo de sesenta folios divididos de la siguiente manera:

- Un texto traducido del árabe al valenciano por Johannes Bonie en $1456^{42}$ ocupa los primeros folios con una veintena de capítulos (ff. $\left.3 r-8 r\right)^{43}$.

- Un texto latino que Johannes Bonie terminó de traducir el doce de marzo de 1464 (ff. 11r-57r) ${ }^{44}$.

- Un texto que supone la traducción parcial del texto valenciano al latín $(57 \mathrm{v}-59 \mathrm{v})^{45}$.

Estos dos últimos testimonios manuscritos parecen de la misma mano. Según las primeras líneas del manuscrito $7416 \mathrm{~A}$, que supone la traducción

conservado sólo una copia manuscrita finalizada en 1483. Esta copia se encuentra actualmente entre los fondos árabes de la Biblioteca de El Escorial, ms. n." 918, Vid. H. Derenbourg y H.P.J. RenAud, Les manuscrits arabes de l'Escurial, Paris, 1941, vol. 1I, p. 24. La edición, traducción y estudio de este tratado fue el trabajo presentado como tesis doctoral por M. Aguiar Aguilar, trabajo que se encuentra inédito. Acerca de CIzz al-Dīn b. Mas ${ }^{\mathrm{c}} \overline{\mathrm{u}}$, autor de este tratado de usos del cuadrante de senos, véase M. AguiAR AculLAR, «En torno a...». En otros trabajos, la que escribe ha dado siempre como título de este tratado Tratado de lo suficiente de la ventaja del uso del cuadrante de senos, y tiene que agradecer la corrección de Samsó acerca de este extremo, que sugería un título más comprensible que el que veníamos usando. Me corrijo, pues, y en principio elijo un híbrido entre mi antigua traducción y la propuesta por Samsó.

41. Acerca de la descripción de estos manuscritos puede verse E. POULLE, «Théorie des planètes...», pp. 129-132.

42. En el ms. $7416 \mathrm{~A}$, ff. $3 \mathrm{v}$ y $58 \mathrm{v}$, dice que el texto se tradujo del árabe al valenciano en esta fecha. "Aquesta es la lectura del xixante trasladada de arabich en lengua vulgar l'any de la nativitat de nostro senyor Deu Iesu Christu 1456 en Paterna horta de Valencia" y "Hec est lectura sexagenarii interpretata ex arabico in linguam uulgarem ualantini ydiomatis anno predicti Domini 1456 in Paterna sic dicta uilla predicte Valentie ciuitatis".

43. "La lectura del xaxante que lo moro alfaqui...".

44. "Plurimorum uirtus ad uirum dumtaxat corpus...".

45. "Lectura sexagenarii quod sarracenus alfachi...". 
incompleta del texto valenciano al latín ${ }^{46}$, el traductor del texto árabe al valenciano es Johannes Bonie, un hombre de gran formación universitaria, maestro en artes y medicina, que pudo ser uno de los médicos del rey René $^{47}$. Por otro lado, el explicit del texto latino contenido en el mismo manuscrito, folio 57r, dice igualmente que este Johannes Bonie es el autor de esta versión ${ }^{48}$. El testimonio manuscrito perteneció después a Oronce Fine, que vivió entre 1494 y 1555, y fue heredado tras su muerte por Antonius Mizaldus, cuya vinculación con el texto aparece dos veces ${ }^{49}$. Al parecer Mizaldus vivió entre los años 1510 y 1578 y fue especialista en previsiones meteorológicas y tratados de jardines.

Mucho menos es lo que sabemos del segundo manuscrito latino $10263^{50}$. Este testimonio ocupa un pequeño trozo que coincide con el contenido de los veinte capítulos que se conservan en el texto escrito en lengua valenciana, pero no supone la traducción de este último. Junto a él está 1475 como fecha, bien de la redacción del texto o bien de la realización de la copia. Al ser un manuscrito tan corto también resulta parco a la hora de explicar los usos y las posibilidades del sexagenarium. Se ocupa sólo del movimiento del sol y las estrellas y deja de lado el problema de la posición de los planetas.

En cuanto a la persona que lo puso por escrito, cabe señalar la posibilidad de que pudiera deberse a Christianus Prolianus ${ }^{51}$, ya que este texto se encuentra en el manuscrito 10263 a continuación de una explicación debida a este autor de cómo combinar los trazados del cuadrante nuevo, del sexagenarium y de los trazados que aparecen generalmente en el dorso del astrolabio. Posiblemente, como señala Poulle 52 , se añadiera un comentario acerca del sexagenarium para aclarar su uso, menos conocido que los otros dos trazados que Prolianus presentaba en el dorso del astrolabio.

En torno al mismo texto poseemos un tercer documento, una impresión realizada por Jean de Guerlins en St.-Pons de Thomières, localidad del de-

46. Folio 57v: "Incipit lectura sexagenarium quam Johannes Bonie ex arabico in ydioma [sic] ualentinum interpretatus est sed quidam in romanam linguam omnium preclarissimam nunc traduxit".

47. Cf. el repertorio del astrólogo Symon de PHARES, Recueil des plus célèbres astrologues et quelques hommes doctes faict par Symon de Phares du temps de Charles VIII'. E. Wickersheimer (ed.), Paris, 1929, p. 260.

48. Folio 57r: "Expletum fuit hoc opus per dominum magistrum Johannem de Bonia ualentinensem 12 mesis marcii $1464^{\prime \prime}$.

49. En los ff. $10 \mathrm{v}$ y $57 \mathrm{v}$. En la primera ocasión reconoce: “...quod tandem hominis mors hereditarium nobis fecit" y su nombre aparece escondido bajo tachaduras, por lo que se adivina con dificultad. En la segunda, su nombre figura en el margen izquierdo como señal de propiedad.

50. En adelante, Lat. 10263. Ocupa los folios 137r-147r. La descripción de este manuscrito se encuentra en E. POULLE, «Théorie des planètes...», pp. 129-130.

51. Ibidem, p. 129.

52. Ibidem. 
partamento de Hérault, Francia, en el año $1516^{53}$. Su autor es Bartholomeus de Solliolis, un reconocido médico e investigador ${ }^{54}$.

Este último testimonio contiene ciento cincuenta capítulos, distribuidos en cuatro partes, que abarcan los siguientes contenidos:

a) Los relativos al movimiento del sol y las estrellas, que coincide con los apartados comprendidos en el manuscrito $7416 \mathrm{~A}$, en la parte escrita en la lengua valenciana, y en el manuscrito Lat. 10263.

b) Los pertenecientes al movimiento planetario ocupan la segunda y tercera parte.

c) Los que conciernen a problemas de geometría práctica.

\section{EL CONTENIDO DE LOS CAPÍTULOS DE LOS TRATADOS DEL SEXAGENARIUM}

Transcribimos a continuación los títulos de los capítulos que componen cada uno de los textos que conocemos relativos al sexagenarium ${ }^{55}$.

Contenido de la versión valenciana del tratado del sexagenarium (Biblioteca Nacional de París, manuscrito latino n. ${ }^{\circ} 7416 \mathrm{~A}$, ff. 3r-8r).

La entrada tracta dels noms del instrument soes lo centre, la circunferencia, lo ffil, lo plom, la linea del lavant, la linea del ponent, les miges cordes.

Lo primer capitol es que mostra com devem pendre la altitut del sol per les horelas ho fforats ab lo plom.

Lo segon capitol tracta en conexer la miga corda del arch he larch do la miga corda.

Lo ters capitol tracta en conexer la hombra de la altitut he la altitut de la hombra.

Lo quart capitol tracta en conexer la declinacio he lo contrari.

53. Vid. Louis DESGRAVEs, Répertoire bibliographique des livres imprimés en France au seizième siècle, Baden-Baden, 1979, $29^{\mathrm{e}}$ livraison (Bibliotheca Bibliographica Aureliana, LXXVI), additions et corrections, p. 119. Según este repertorio, hay un ejemplar de esta edición en París, en la Mazarina, bajo la signatura Rés. 37975. Por el momento, no nos ha sido posible consultar esta impresión.

54. Una nota autógrafa de Oronce Fine que presenta el manuscrito $7416 \mathrm{~A}$ en el f. $10 \mathrm{v}$ hace mención a que Bartholomeus de Solliolis realizó su obra en 1500: "...curauit impressos Bartholomeus de Solliolis Viuariensis medicus usque ad numerum 150 in ciuitate Diui Pontii Thomerarum anno Christi 1500...".

55. Thorndike ya ofreció en su día la transcripción de los títulos o cánones de los distintos textos latinos. No hay transcripción suya publicada de los títulos de la versión valenciana. Vid. L. THORNDIKE, «Sexagenarium...», pp. 130-133. Nuestra transcripción añade estos últimos más algunas diferencias respecto a la lectura de Thorndike. 
Lo quint capitol es conexer lo pol he la altitut del migiorns he de la declinacio.

Lo sex capitol es en conexer la rael absoluta he la altitut del diametra he la reel adobada so es la absoluta. del arch.

Lo septeme capitol es ha conexer la mixtat del creximent he la mixtat

Lo huyteme capitol es ha conexer lo pasat he lo restant del dia.

Lo noneme capitol es en conexer lo moviment de la rodo entre les oracions dels moros so es la oracio de migiorn, que la nomenen adoactri he laltra oracio ha nom hatar.

Lo deseme capitol es en conexer la amplura de la alba he apres del sol post.

Lo onzeme capitol es en conexer la amplura del lavant. azimuts.

Lo dozeme capitol es en concexer la altitut que no ha en ella dita altitut

Lo trezeme capitol es en conexer los azimuts.

Lo quatorseme capitol es en conexer lo azimuth per a mecha.

Lo quizema capitol es en conexer les quatre parts del mon com les trauras a la part de mecha. blit.

Lo sexzeme capitol es en conexer les absencions del sercle dret ho ob-

Lo deseteme capitol es en conexer la hobra de la stela he hobrar ab la stella. sabut.

Lo dishuyteme capitol es en conexer lo stament de la stella en temps

Lo desenoneme capitol es en conexer tota alitut sobre la terra.

Lo vinteme capitol es en conexer la amplura dels rius ho ffondura de pous.

Contenido de la traducción latina inacabada de la versión valenciana del tratado del sexagenarium (Biblioteca Nacional de París, manuscrito latino n..$^{\circ} 7416 \mathrm{~A}$, ff. $57 \mathrm{v}-59 \mathrm{v})^{56}$.

Ingressus huius libri est de nominibus instrumenti et docentur, secundum centrum, circunferentia, filum, plumbum, linea orientis, linea occidentis et medie corde.

Primum caput docet quomodo capienda est solis altitudo per auriculas instrumenti aut foramina cum filo in quo plumbum est.

Secundum caput docet mediam cordam ex arcu et arcum ex media corda.

56. Ibidem, p. 132. 
Tertium caput umbram ex altitudine docet et altitudinem ex umbra demostrat.

Quartum caput docet cognoscere declinationem et econtrario.

Quintum caput polum docet et declinationis altitudinem in meridie.

Sextum docet absolutam radicem et diametri altitudinem atque radicem equatam.

Septimum eius caput docet augmenti medietatem et arcus medietatem.

Octałum caput docet transactam diem partem et futuram.

Nonum autem caput cognoscit motum rote inter serracenorum orationes hoc est inter orationem meridiei que ab eis nuncupatur adohari et alia dicitur athar.

Decimum enim caput docet crepusculum matutinum et uespertinum.

Undecimum caput manifestat nobis latitudinem orientis.

Duodecimum caput est $[\mathrm{ad}]^{57}$ cognoscendum altitudinem que in dicta altitudine $[a z i m u t h]^{58}$ non habet.

Caput 13 manifestat nobis azimuths.

Caput autem 14 docet cognoscere azimuth versus Mecam.

Quintum decimum enim caput docet quatuor mundi plagas quomodo eas duces ad partes Mesce.

Caput 16 docet ascensiones rectas aut obliquas signorum.

Caput 17 manifestat operationem per stellam fabricandam.

Caput 18 est ad cognoscendum statum stelle in tempore scito.

Caput 19 docet quamcumque altitudinem supra terram.

Caput 20 et ultimum manifestat nobis latitudinem fluuiorum atque puteorum profunditatem.

Contenido de la versión latina en veinticinco capítulos (Biblioteca Nacional de París, manuscrito latino n. ${ }^{\circ} 10263$, ff. $\left.141 \mathrm{v}-147 \mathrm{r}\right)^{59}$.

Canon secundus ${ }^{60}$, de corda recta uersa aut media et eorum sagitta cognoscenda.

Canon tertius, de uero loco solis inuestigando.

Canon quartus, de declinatione cuiuslibet gradus zodiaci ab equatore.

Canon quintus, de altitudine solis in meridie inuenienda.

57. Suponemos que antes de cognoscendum debe aparecer la preposición ad aunque no aparezca en el manuscrito.

58. Suponemos de igual forma que debe entenderse que falta la palabra azimuth.

59. Vid. L. THORNDIKE, «Sexagenarium...», p. 133.

60. El capítulo primero no tiene título y consiste en unas breves líneas que describen el instrumento. 
Canon sextus, si arcum diurnum et nocturnum gradu zodiaci cuiuscumque uelis inuestigare.

Canon septimus, de distancia horarum equalium a meridie similiter et magnalium cognoscendo.

Canon octauus, de horis preteritis ab ortu solis equalium uel inequalium demonstrandis.

Canon decimus, de altitudine uel per horas ostendere.

Canon undecimus, de horis inequalibus ad equales reducere.

Canon 12, de ascensionibus signorum in circulo recto inuestigandis.

Canon 13, de signorum ascensionibus in circulo obliquo precise.

Canon 14, de quantitatis zodiaci ascendentis cum circulo recto inuenienda.

Canon 15, de quantitate zodiaci que simul cum equinoxiali ascendit in spera obliqua habenda.

Canon 16, de gradu ascendentis et principia quatuor domorum inueniendis.

Canon 17, de gradibus ascendentis inuenire

Canon 18, de principiis 12 domorum habendis.

Canon 19, de latitudine ortus et occaus solis inuenienda.

Canon 20, de arcu horarum ab ortu solis in circulo zenith inueniendo.

Canon 21, de noticia partis azimuth solis cuiuscumque hore in quartis sole in septentrionalibus signis existente. bus.

Canon 22, est istud de parte azimuth cuiuscumque hore in meridionali-

Canon 23, de latitudine habenda cuiusque stelle fixe in celo.

Canon 24, de declinationibus fixarum stellarum et eorumdem maioribus altitudinibus cognoscendis.

Canon 25, de arcus nocturnus unicuiusque stelle manifestatione.

Contenido de la versión latina de cuarenta y seis capítulos repartidos en cinco partes (Biblioteca Nacional de París, manuscrito latino ${ }^{\circ} 7416 \mathrm{~A}$, ff. $1 \mathrm{r}-57 \mathrm{r}$.

Prime partis

Capitulum primum, tam instrumenti quam partium eiusdem.

Capitulum secundum, de arcuum cordarumque et sagitarum inuencione.

Capitulum tertium, ad inueniendum altitudinem stellarum atque aliarum rerum.

Capitulum quartum, de loco solis uero inueniendo cum hoc instrumento.

Capitulum quintum, ad habendum declinacionem solis ab equinocciali. 
Capitulum sextum, de equacione diametri habenda.

Capitulum septimum, de noticia arcus diei et noctis.

Capitulum octauum, de altitudine solis in meridie habenda.

Capitulum nouenum, ad sciendum horas tam equales quam inequales.

Capitulum decimum, de altitudine solis per horas habenda.

Capitulum undecimum, de modo inueniendi azimuth solis in orizonte.

Capitulum duodecimum, ad sciendum tempus per quod sol stat in qualibus quartarum principalium.

Capitulum 13, ad sciendum partem azimuth solis in qualibet quartarum principalium in diebus maioribus suis noctibus.

Capitulum 14, ad sciendum partem azimuth solis in diebus minoribus suis noctibus.

Capitulum 15, ad sciendum ascensiones signorum in circulo recto.

Capitulum 16, de noticia ascensionis signorum in circulo obliquo.

Capitulum 17, de noticia gradu zodiaci qui conditur cum aliqua parte equinocciali tam in recta quam in spera obliqua. domus.

Capitulum 18, de noticia ascendentis gradus qui principium est prime

Capitulum 19, de noticia principiorum aliarum domorum a prima.

Capitulum 20, ad sciendum tantum principia quatuor domorum principalium.

Capitulum 21, de habenda declinatione uniuscuiusque stelle ab equinocciali.

Capitulum 22, de noticia arcus uniuscuiusque stelle habenda.

Capitulum 23, de noticia hore ascensus uniuscuiusque stelle.

Capitulum 24, ad sciendum horas in nocte cum stellarum fixarum.

Secunde partis

Capitulum primum, de modo faciendi dorsum huius instrumenti.

Capitulum secundum, de modo operandi per dorsum huius instrumenti.

Capitulum tertium, de modo equacionis octaue spere ex augium habenda.

Capitulum quartum, de noticia eorum que presupponuntur ad equationes 7 sperarum inferiorum habenda.

Capitulum quintum, de noticia equacionis centri in tribus superioribus et uenere habenda.

Capitulum septimum ${ }^{61}$, de noticia equationis centri in luna habenda.

61. Falta el capítulo sexto. Thorndike suponía que el comienzo de dicho capítulo había que situarlo dentro del texto en el f. $34 \mathrm{v}$. 
Capitulum octauum, de noticia equationis argumenti in omnibus planetis postquam in sole habenda.

Incipit tertia pars de noticia umbrarum

Capitulum primum, de noticia punctorum umbre tam recte quam uerse habenda.

Capitulum secundum, de noticia compositionis zelindi habenda. da.

Capitulum tertium, de notica compositionis horarum horalogii haben-

Capitulum quartum, de noticia compositionis horologii in plano habentis solum rectum habenda.

Capitulum quintum, de noticia compositionis horologii in plano habenti filum obliquum.

Capitulum sextum, de noticia compositionis horologii quod sub fforma anuli ponitur habenda. habenda.

Capitulum septimum, de noticia mensure altitudinis rei per umbram

Incipit quarta pars de operationibus geometricis benda.

Capitulum primum, de noticia mensurarum in rebus accesibilibus ha-

Capitulum secundum, de noticia mensurarum in rebus inaccesibilibus habenda.

Incipit quinta pars de operationibus arismeticis

Capitulum primum, de quibusdam presuppositis ad huius operationes.

Capitulum secundum, de noticia multiplicationis habenda.

Capitulum tertium, de noticia diuisionis habenda.

Capitulum quartum, de noticia inuentionis numeros in aliqua proportione habenda. meris.

Capitulum quintum, de noticia inuentionis radicem quadratam in nu-

\section{CONCLUSIONES}

Este trabajo supone, en primer lugar, un acercamiento previo a la bibliografía relativa al sexagenarium, que es muy escasa y reiterativa, tomando como punto de partida el estudio que Poulle realizó en 1966. Se trata de un extenso artículo en el que ofrecía algunos datos en relación a la historia de los manuscritos y un análisis del uso del instrumento. 
También hemos hecho un primer acercamiento a los manuscritos conservados relativos al tratado del sexagenarium, primero a través de microfilmes y después de forma directa en París.

Nuestro principal objetivo, del que estas páginas son un avance, es la edición de los manuscritos que contienen el tratado del sexagenarium, debido fundamentalmente a dos circunstancias: al hecho de que los trabajos posteriores al de Poulle no contengan aportaciones nuevas, y a que no se haya llevado a cabo hasta la fecha la confrontación de los manuscritos de la Biblioteca Nacional de París con la finalidad de editarlos convenientemente.

Asimismo, tras una primera lectura y transcripción de la versión valenciana y de la latina breve, contenidas ambas en el manuscrito $7416 \mathrm{~A}$, creemos que es necesario hacer las siguientes precisiones:

1. El título del tratado árabe del sexagenarium no es Archycala Ali, como se entiende por la transcripción que Poulle hizo de la nota que escribió Mizaldus en el primer folio del texto del manuscrito $7416 \mathrm{~A}^{62}$. Debe entenderse, tal y como señalan los diferentes textos de este misceláneo -el valenciano y su versión latina incompleta, y el texto latino completo- que el tratado árabe del sexagenarium es mencionado como la risāla de ${ }^{c} A l \bar{l}$, "el tratado de "Alí". Otra cuestión es el problema de identificar a ese "Alī y a su risäla, aspecto éste que no vamos a tocar aquí.

2. Al hilo de esto, creemos que la denominación de sexagenarium para el instrumento sobre cuyos usos versan los distintos tratados podría provenir de la traducción del árabe sittūni , adjetivo que en ocasiones se encuentra para hacer referencia al cuadrante de senos sexagesimal. En efecto, la versión valenciana del tratado del sexagenarium parece simplemente una traducción de un tratado árabe breve sobre los usos del cuadrante de senos. En ningún momento se hace mención en esta versión a la llamada "cara planetaria". Obviamente, lo mismo ocurre con su traducción latina inacabada. Pensamos que habría que entender que el "tratado del sexagenarium" es sencillamente un "tratado de usos del cuadrante de senos sexagesimal", al menos en el caso de la versión valenciana y su traducción latina inacabada, que nos ha llegado de una forma interesantísima: del árabe a una lengua vernácula, el valenciano, y de ésta al latín.

62. Véase E. POULLE, «Théorie des planètes...», p. 132, nota 10, donde transcribe la nota de Mizaldus que encabeza el texto latino del manuscrito 7416A de la Biblioteca Nacional de París. Poulle transcribió el comienzo del folio $10 \mathrm{v}$ de este manuscrito como "Hic liber arabice uocatur Archicala Aly, latine Sexagenarium instrumentum". Creemos que debe leerse "Hic liber arabice uocatur Arrhicala Aly, latine sexagenarium instrumentum". La versión parcial en valenciano y su traducción inacabada al latín corroboran nuestra lectura, ya que dicen así: "Ye ya non lo primer libre en lo propri arabich arricala de haly" (ms. 7416A, f. 3v) y "et iste liber in arabico arrichala aly" (ms. 7416A, f. 58v). Thorndike leyó arrhicala aly, cf. «Sexagenarium...., p. 130. 
Las cosas son distintas si analizamos el contenido de los otros dos textos latinos. Son textos con muchos más capítulos, su distribución es completamente diferente y su contenido también. Por todo ello nos preguntamos hasta qué punto podemos establecer una relación textual entre los cuatro testimonios manuscritos, y entre éstos y el instrumento de Oxford. Estimamos que los otros dos testimonios manuscritos contienen ya mezcla de otros contenidos y que quizás, tras una reclasificación, habría que considerarlos como pertenecientes a una tradición textual diferente. Los cuatro casos sí que creemos que convergen en un punto común: el cuadrante de senos. Puede que tengamos algunas respuestas tras la edición, traducción y estudio de los textos, trabajo que nos planteamos realizar a largo plazo.

3. En cualquier caso, el establecimiento del stemma codicum presentaría cierta dificultad ya que tenemos distintas versiones en dos lenguas diferentes -el valenciano y el latín- que no son en ningún caso copias entre sí, lo que impediría determinar los puntos divergentes que permitirían situar cada testimonio manuscrito en su lugar. No obstante, planteamos dos stemma hipotéticos, de los que uno -o ninguno- será el definitivo, una vez que hayamos editado la totalidad de los testimonios manuscritos. Estas hipótesis se basan en nuestro primer acercamiento a los tratados conservados acerca del sexagenarium y son las siguientes.

a) El stemma codicum podría tener, a partir de un arquetipo $\omega$ en lengua árabe, dos ramas. Una de ellas contiene primero la versión valenciana copiada en 1463 de la de Johannes Bonie de 1456, en la que se recogen sólo veinte capítulos de la obra original. De esta versión valenciana se hizo una traducción latina que no llegó a acabarse y que se detiene nada más comenzar el canon tercero.

La otra rama del stemma contiene la traducción completa al latín del tratado árabe hecha por Johannes Bonie en 1464. De ella podría derivar la versión latina breve de 1475 que se nos ha conservado en el manuscrito latino 10263.

$\omega$



Se da la circunstancia de que tanto la traducción inacabada al latín de la versión valenciana -manuscrito $7416 \mathrm{~A}$ - como la traducción completa al latín del tratado árabe de 1464 se deben a la misma mano. Por otro lado, del comienzo de la traducción al latín de la versión valenciana podría deducirse que el texto valenciano fue obra de Johannes Bonie y también que alguien 
hizo una traducción al latín del tratado del sexagenarium, bien a partir del árabe o bien a partir del valenciano ${ }^{63}$.

Estos tres elementos nos llevaron a proponer un segundo stemma hipotético, que es el siguiente.

b) El stemma tendría dos ramas que derivarian de un arquetipo $\omega$ en lengua árabe. Una de ellas contendría la versión valenciana de 1463, con un antecedente $\alpha$ de 1456, y la traducción inacabada al latín también conservada en el manuscrito $7416 \mathrm{~A}$. La otra rama se abriría con un antecedente latino $\beta$, no conservado y totalmente hipotético, cuya existencia imaginamos a partir de los tres elementos antes expuestos. Derivando de $\beta$ tendríamos primero la versión latina completa del tratado árabe de 1464 , que se encuentra en el misceláneo de sesenta folios - manuscrito $7416 \mathrm{~A}-\mathrm{y}$, luego, la versión latina breve de 1475 que se nos ha conservado en el manuscrito latino 10263.

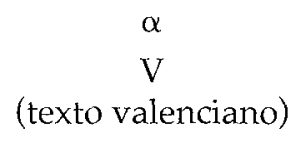

$\omega$

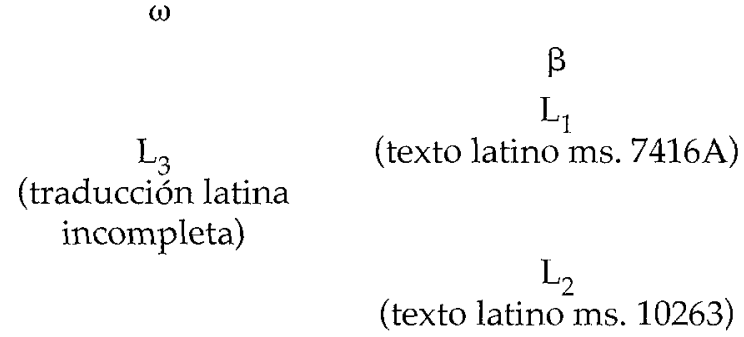

\section{RESUMEN}

Este trabajo supone un primer acercamiento a la bibliografía y a los manuscritos relativos al tratado del sexagenarium. Nuestro principal objetivo es la edición de dichos manuscritos debido al hecho de que los trabajos posteriores al de Poulle (1966) no contienen aportaciones nuevas. A esto se añade que no se ha llevado a cabo hasta la fecha la confrontación de los manuscritos de la Bibliothèque Nationale de París con la finalidad de editarlos convenientemente.

\section{ABSTRACT}

This paper is supposed to be an approach to the bibliography and manuscripts related to the sexagenarium treatise. Our main goal is to edit these

63. Manuscrito $7416 \mathrm{~A}$, f. $57 \mathrm{v}$. 
manuscripts since all the studies carried out after Poulle's work (1966) have not made any contribution. It should be added the fact that, up to the present, no comparison has been made of the manuscripts of the Bibliothèque Nationale of Paris and, for this reason, it has not been possible to edit them properly. 